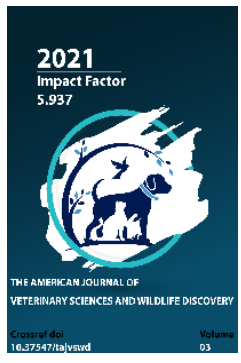

Journal Website: http://theamericanjour nals.com/index.php/taj vswd

Copyright: Original content from this work may be used under the terms of the creative commons attributes 4.0 licence.

\section{Chemical Therapy Method Of Breast Tumors In Dogs In Samarkand}

Yulchiev J.B.

PhD, Assistant, Samarkand Institute Of Veterinary Medicine, Uzbekistan

Mirsaidova R.R.

Assistant, Samarkand Institute Of Veterinary Medicine, Uzbekistan

\title{
ABSTRACT
}

This article presents the results of scientific research on the etiology, clinical signs and chemotherapy treatment of breast tumors among dogs.

\section{KEYWORDS}

Breast cancer, estrogen hormones, chemotherapeutic agent, cyclophosphamide, anthropogenic factors, nitrogen mustard, lymphogranulomatosis, radiation therapy, cryodestruction and general hyperthermia.

\section{INTRODUCTION}

One of the most common pathologies among dogs today is tumor pathology, where many different pathological processes, including breast diseases, slow down their vital functions, reduce their activity, force them to write off, and in some cases even lead to their death [3].

The spread of tumors among dogs is as common as in humans. In terms of anatomical structure, it is no coincidence that breast tumors in dogs are in the forefront today $[1,2]$. The large number of mammary glands in dogs compared to other animals, differences in the activity of the circulatory and lymphatic systems in the mammary glands and, of course, the specificity of the course of the sexual cycle ensure that dogs have a high risk of breast cancer [4]. One of the main reasons for the prevalence of breast tumors among dogs and the serious complications of the disease are anthropogenic factors, i.e. indifference of dog owners, sudden increase in tumor size due to untimely consultation with veterinarians and deterioration of the 
general condition of dogs. Therefore, the study of breast diseases in dogs, including breast tumors in dogs, the use of chemotherapy in their effective treatment is relevant [5].

\section{OBJECT AND METHOD OF RESEARCH}

The research was conducted in the clinic of the Department of Veterinary Surgery and Obstetrics, Faculty of Veterinary Prevention and Treatment, Samarkand Institute of Veterinary Medicine. The object of the study was dogs with breast cancer and their blood samples, tumor tissue. The purpose of the study is to study the etiopathogenesis of breast diseases in dogs in Samarkand, the use of accurate and early diagnosis of the disease and the improvement of treatment methods.

\section{ANALYSIS OF THE OBTAINED RESULTS}

As a result of experiments on breast tumors and dispensaries on the spread of tumors in dogs, the occurrence of tumors among dogs in our country was observed in dogs aged 3 to 14 years, the highest incidence was recorded in dogs aged 6-9 years. Clinical studies and anamnestic data have shown that $75-80 \%$ of dogs with breast cancer are not born at all or have given birth once or twice in their lifetime.

Experiments have indeed revealed that sexual dysfunction in female dogs, the development of vascular hyperemia and glandular tissue hyperplasia as a result of years of chronic tickling of breast hormones by sex hormones, leads to the subsequent formation of tumors. At the same time, our studies have shown that a sharp increase in the size of the tumor as a result of negligence of the owners of dogs in the population, its progressive growth and only at the stage of transition of the tumor from local pathology to general pathology.
The use of various hormonal drugs to protect dogs from pregnancy also increases their risk of developing breast cancer.

In veterinary oncology, the surgical method is used as the main method of treatment. Conservative methods of tumor treatment include chemotherapy, hormone therapy, immunotherapy, radiation therapy, cryodestruction, and general hyperthermia.

Nowadays, anti-tumor chemotherapy as well as surgical methods are widely used, mainly in the preoperative process or in cases of metastasis, as a method of postoperative treatment to control the growth of tumor tissue.

Malignant tumor chemotherapy is one of the most important treatments in oncology, based on the use of various chemicals against malignant tumors. The history of modern chemotherapy dates back to 1946. At the same time, for the first time a strong poison nitrogen mustard was used to treat lymphogranulomatosis.

From time immemorial, people have tried to treat cancer with various substances. They were plant, animal products, minerals, and so on. Their choice was empirical, that is, there was no scientific basis. It is important to determine the effectiveness of treatment, the routes of administration of anti-tumor drugs and chemotherapy in combination with other treatments (surgery, radiation therapy).

There are systemic, regional and local types of drug delivery. Systemic chemotherapy is the delivery of drugs into the bloodstream by all means in order to have a general effect during the dissemination (spread) of tumors. Local chemotherapy is the application of a chemical drug in the form of an ointment or liquid 
directly to the tumor (intraural), intravenous (pleural, intra-abdominal, 1st vesicle, etc.) or intramural.

Experimental dogs were treated with postoperative chemotherapy, in which anticancer drugs were administered orally once every 2 days for 15 days at a dose of $0.4 \mathrm{~g}$ (6 mg / kg) of cyclophosphamide, a drug that inhibits protein synthesis in tumor cells.

Cyclophosphamide is a white, crystalline powder that is an anti-tumor agent that can be administered orally and parenterally, and is chemically close to the nitrogen analogue mustard. The drug has cytostatic and immunodepressive effects. It breaks down tumor cells directly through components that are active in the phosphatase process, and the protein molecules of the cell attack the nucleophilic centers, disrupting DNA and RNA synthesis in them and blocking the process of mitotic division. The drug is mainly used in acute and chronic lymphoblastic, lymphocytic, myeloid and myelogenous leukemias; in malignant lymphoma, plasmocytoma and their metastases, ovarian, lung cancer, all types of breast tumors, neuroblastomas, sarcomas and osteosarcomas; recommended for use in progressive "autoimmune diseases": pathologies such as rheumatoid arthritis, psoriatic arthropathy, scleroderma and systemic vasculitis.

In addition, as an additional anti-tumor chemical, the ASD-2 stimulant was given to dogs orally 25 drops per day on an empty stomach for half a hour before meals.

At the end of the experiment, the use of this drug reduces the healing of surgical wounds by 3-5 days, reduces the symptoms of inflammation in the wound, reduces fluid in the tumor and colloids as a result of recovery of blood and lymphatic circulation, acidosis, proteolysis and phagocytosis of the wound cavity. the formation of granular tissue on the surface and the acceleration of scar formation were observed.

The general condition of the dogs was satisfactory, body temperature was $37-380 \mathrm{C}$, respiration rate was $20-22$ beats / $\mathrm{min}$, heart rate was $75-80$ beats / $\mathrm{min}$.

Also, the negative effects of tumor tissue and cells on the body were not felt at all. No metastases or relapses were observed in sick dogs.

\section{CONCLUSION}

1. In Samarkand, the prevalence of all types of tumors among dogs in the population is $5-7 \%$, of which $35-40 \%$ are breast tumors.

2. In the conditions of the republic, the incidence of cancer occurs in dogs of all ages, and the highest incidence is mainly in dogs 5-10 years old.

3. The main etiological factor in the origin of breast tumors is the tickling of chronic glandular tissue by sex hormones as a result of impaired reproductive function in dogs.

4. The main method of treatment of breast tumors is surgery, in which mastectomy is performed.

5. In addition to the surgical method in the treatment of breast tumors, the use of chemotherapeutic methods gives good results and prevents the occurrence of metastases and relapses.

\section{REFERENCES}

1. L.F. Loseva, F.V. Donenko, O.V. Lebedinskaya. Some pharmacodynamics of cyclophosphamide in experimental 
animals // Medical immunology. - 2011, T.

13. № 4-5. - S. 52.

2. Cyclophosphamide enhances immunity by modulating the balance of dendritic cell subsets in lymphoid organs / T. Nakahara, H. Uchi, A. M. Lesokhin // Blood. - 2010. Vol. 115, №22. - P. 4384-4392.

3. Kalishyan M.S., Sedakova L.A., Andronova N.V. «Possibilities of proved neoad'yuvantnoy polychemotherapy of cancer of the mammary gland with the use of the catalytic system teraftal + ascorbic acid» // RBJ.-2007 g.-t.6.-№1.str.33.

4. Perevodchikova N.I. «Manual for chemotherapy of opholevyx diseases» // Prakticheskaya meditsina, M., 2005, p.698.

5. Yakunina M.N, Treshchalina E.M., Shimshirt A.A. «Perenosimost sobakami i koshkami chemiotherapy s taxoterom pri rake molochnoy jelezy».// RVJ, 2010 g. №2.-str.12-15. 